\title{
A Novel Method for Cortical Sulcal Fundi Extraction
}

\author{
Gang $\mathrm{Li}^{1,2}$, Tianming $\mathrm{Liu}^{1}$, Jingxin $\mathrm{Nie}^{2}$, Lei Guo ${ }^{2}$, and Stephen T.C. Wong ${ }^{1}$ \\ ${ }^{1}$ The Center for Biotechnology and Informatics, The Methodist Hospital Research \\ Institute and Department of Radiology, The Methodist Hospital, Weill Medical College \\ of Cornell University \\ ${ }^{2}$ School of Automation, Northwestern Polytechnic University, Xian, China
}

\begin{abstract}
Sulcal fundi are 3D curves along the bottom of sulcal regions of the human cerebral cortex. In this paper, we propose a novel automatic method for extraction of sulcal fundi from triangulated cortical surface. Compared to existing methods, the proposed method can find accurate sulcal fundi using curvatures and curvature derivatives without manual interaction. Given a triangulated cortical surface, our method is composed of four steps: estimating curvatures and curvature derivatives for each vertex, detecting the sulcal fundi segments in each triangle, linking the sulcal fundi segments and combining of adjacent sulcal fundi, and connecting breaking sulcal fundi and smoothing using the fast marching method on the cortical surface. The proposed sulcal fundi extraction method is applied to ten normal brain inner cortical surfaces. We quantitatively validated the proposed method of sulcal fundi extraction using manually labeled sulcal fundi by experts as the ground truth.
\end{abstract}

\section{Introduction}

The human cerebral cortex is a highly convoluted structure composed of sulci and gyri, corresponding to the valleys and ridges on cortical surface respectively. Sulcal fundi are 3D curves along the bottom of sulcal regions of human cerebral cortex. Major sulci and gyri are common anatomical landmarks in human brains, even though the pattern of sulci and gyri geometry could be quite different across individuals [1]. Thus, major sulci have been extensively used for assisting deformable registration of MR brain images and analyzing the variation of healthy human brain, as well as differentiating the difference between normal brain and diseased ones. Since it is extremely time consuming to label sulci manually, automation has been actively investigated. Methods have been proposed for sulci or sulcal fundi extraction either on MR volumetric images [2, 4, 9] or constructed cortical surfaces [3, 5, 6, 7, 8, 9, 13]. Skeletization or thinning based methods $[2,4,6,8]$ and curve tracking based methods $[3,13]$ are among the most commonly used techniques for sulci or sulcal fundi extraction. However, applying skeletization or thinning based methods onto the cortical surface cannot assure that the extracted sulcal fundi are unbiased from the true sulcal fundi, as the sulcal regions might not be symmetric around sulcal fundi. On the other hand, in curve tracking based methods, start or end points of sulcal fundi have to be manually selected. Thus, computational methods for finding sulcal fundi in an automated and accurate manner are much needed. 


\section{Method}

\subsection{Overview}

In this paper, we propose a novel automatic method for extraction of sulcal fundi from triangulated cortical surface. Compared to existing methods, the proposed method can automatically find the accurate sulcal fundi using curvatures and curvature derivatives. Given a constructed triangulated cortical surface, our method of sulcal fundi extraction is composed of four steps, as summarized in Figure 1. Firstly, we adopt the finite difference method to estimate the principal curvatures and directions and the curvature derivatives along the principal directions for each vertex. Then we detect the sulcal fundi segments in each triangle based on the curvatures and curvature derivatives. Afterwards, we link the sulcal fundi segments into continuous sulcal fundi curves and combine adjacent sulcal fundi curves caused by numerical estimation errors. Finally, we connect the breaking sulcal fundi curves and smooth bumping sulcal fundi by applying the fast marching method on the cortical surface.

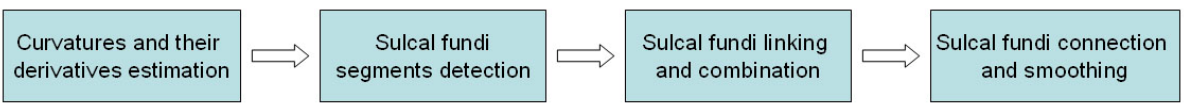

Fig. 1. Flow chart of the sulcal fundi extraction method

\subsection{Estimating Curvatures and Curvature Derivatives}

Curvatures and their derivatives are fundamental properties for cortical surface analysis. Herein, we adopt a robust method to estimate principal curvatures, principal directions, and curvature derivatives along principal directions as described in [10].

\subsection{Detection of Sulcal Fundi Segments}

To determine whether each triangle contains sulcal fundi segments or not, we adopt a similar method describe in [11], which is originally developed for ridge and valley detection on mesh and resembles to work in [16]. Given a triangulated cortical surface denote the maximum principal curvature be $c_{\max }$ (the curvature with maximum absolute value in the two principal curvatures) and the corresponding principal direction be $\mathbf{p}_{\max }$. Denote $d_{\max }=\partial c_{\max } / \partial \mathbf{p}_{\max }$ as the directional derivative of $c_{\max }$ along with $\mathbf{p}_{\max }$. The criterion for sulcal fundi segment detection is formulated as:

$$
c_{\max }<0, d_{\max }=\partial c_{\max } / \partial \mathbf{p}_{\max }=0, \partial d_{\max } / \partial \mathbf{p}_{\max }>0
$$

It means that sulcal fundi should be at the location where $c_{\max }$ is negative and the first order directional derivative of $c_{\max }$ vanishes (zero-crossing of $d_{\max }$ ), and the second order directional derivative of $c_{\max }$ reaches positive values. Given a triangle in a cortical surface (denoting the three vertices as: $\mathbf{v}_{1}, \mathbf{v}_{2}$ and $\mathbf{v}_{3}$ ), we check whether the 
triangle contains sulcal fundi segments by inspecting whether the three edges contain sulcal fundi points. Without loss of generality, we use the edge formed by $\mathbf{v}_{1}$ and $\mathbf{v}_{2}$ as an instance. Firstly, we inspect $c_{\max }$ at vertices $\mathbf{v}_{1}$ and $\mathbf{v}_{2}$ to make sure:

$$
c_{\max }\left(\mathbf{v}_{1}\right)<0 \text { and } c_{\max }\left(\mathbf{v}_{2}\right)<0
$$

Then, we check whether the edge contains the first order directional derivative of $c_{\max }$ vanished point with the following formula:

$$
d_{\text {max }}\left(\mathbf{v}_{1}\right) \mathbf{p}_{\text {max }}\left(\mathbf{v}_{1}\right) d_{\text {max }}\left(\mathbf{v}_{2}\right) \mathbf{p}_{\text {max }}\left(\mathbf{v}_{2}\right)<0
$$

Then, to make sure that the edge contains true sulcal fundi point in theory, the maximum principal curvature decreases along the edge should satisfy:

$$
d_{\text {max }}\left(\mathbf{v}_{1}\right) \mathbf{p}_{\text {max }}\left(\mathbf{v}_{1}\right)\left(\mathbf{v}_{2}-\mathbf{v}_{1}\right)<0 \text { or } d_{\text {max }}\left(\mathbf{v}_{2}\right) \mathbf{p}_{\text {max }}\left(\mathbf{v}_{2}\right)\left(\mathbf{v}_{1}-\mathbf{v}_{2}\right)<0
$$

Herein, we loose the conditional checks. If the edge passes the above three conditional checks, a strict sulcal fundi point is found in the edge. Otherwise, if the edge only passed the first two conditional checks, a candidate sulcal fundi point is found. Once a sulcal fundi point is found, we calculate the exact unbiased position of the sulcal fundi point with the linear interpolation method [11]:

$$
\mathbf{v}=\frac{\left|d_{\text {max }}\left(\mathbf{v}_{1}\right)\right| \times \mathbf{v}_{2}+\left|d_{\text {max }}\left(\mathbf{v}_{2}\right)\right| \times \mathbf{v}_{1}}{\left|d_{\text {max }}\left(\mathbf{v}_{1}\right)\right|+\left|d_{\text {max }}\left(\mathbf{v}_{2}\right)\right|}
$$

After checking the three edges in the triangle, if two of the three edges have sulcal fundi points, we connect the two points as a sulcal fundi segment; otherwise if all of the three edges contain sulcal fundi points, we connect the three vertices to the center of the triangle to form three sulcal fundi segments, which might correspond to the location of intersection of the sulcal fundi curves. Afterwards, we distinguish the segments as strict or candidate sulcal fundi segments. If a sulcal fundi point in a segment is a candidate point, we consider the segment as a candidate sulcal fundi segment, or a strict segment otherwise. Figure 2 shows an instance of sulcal fundi segments detection in a cortical surface.

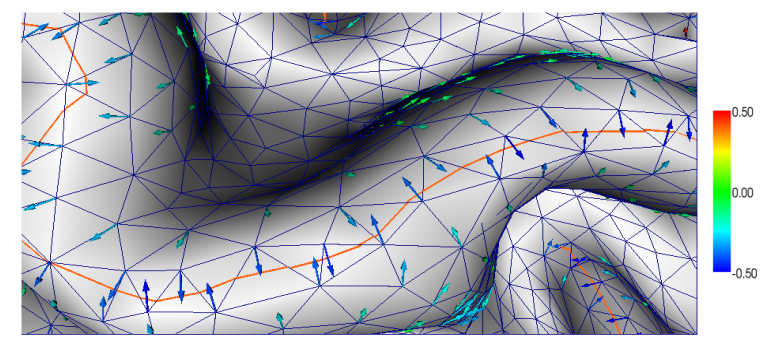

Fig. 2. An instance of sulcal fundi segment detection in a cortical surface. The orange curves represent the sulci fundi segments. The arrow at each vertex indicates the maximum principal direction and the colors of the arrows indicate the values of the maximum principal curvatures. 


\subsection{Linking Sulcal Fundi Segments}

With the detected sulcal fundi segments, we link them into continuous sulcal fundi curves. We distinguish the sulcal fundi segments into strict and candidate segments, because we observe sometimes candidate segments can connect with strict segments to form a continuous sulcal fundi curve. If we only use strict segments, the sulcal fundi will be interrupted around candidate segments. Starting from a strict segment, a sulcal fundi curve is formed by adding adjacent segments that shares the same edge or center with the current segment. After linking we obtain a set of sulcal fundi curves. Due to the error of numerical estimation, sulcal fundi around junction regions are often interrupted. To deal with this situation, we check one ring neighborhood of each vertex. If two or more different sulcal fundi are in the neighborhood and $c_{\max }$ at the vertex is negative, we combine these sulcal fundi together.

\subsection{Connection of Breaking Sulcal Fundi and Smoothing}

After linking the sulcal fundi segments and combining adjacent sulcal fundi, we obtained a series of continuous sulcal fundi curves. Some sulcal fundi may be very short, which correspond to the breaking sulcal fundi or inherently short, minor sulcal fundi. Thus we have to connect breaking sulcal fundi together, however, we may not know which ones are interrupted and which ones are inherently short. To deal with this problem, we adopt the fast marching method on triangulated surface [12]. From each ending point of the extracted sulcal fundi curves, we search in a geodesic region ( $8 \mathrm{~mm}$ as the geodesic threshold) to find out whether other sulcal fundi are in the region. Once other sulcal fundi curves are found, we tentatively connect the end point to the found sulcal fundi by computing the weighted geodesic path on the surface using fast marching method. As the sulcal fundi are located at regions with large negative $c_{\text {max }}$, we set the marching speed at vertex $\mathbf{x}$ as follows:

$$
F_{1}(\mathbf{x})=\left\{\begin{array}{l}
1.0, \text { if }\left(\mathrm{c}_{\max }(\mathbf{x})<T\right) \\
e^{\alpha}, \text { if }\left(\mathrm{c}_{\max }(\mathbf{x})>-T\right) \\
e^{\alpha\left|\mathrm{c}_{\max }(\mathbf{x})-T\right|}
\end{array}\right.
$$

where $T$ is a curvature threshold parameter and $\alpha$ is a weighting parameter. This formula makes sure the sulcal root regions have faster marching speeds, and gyral crown regions have slower marching speeds. We set $T=-1.0$ and $\alpha=-5.0$ throughout the paper. However, the geodesic path may wrongly connect two inherent separated sulcal fundi by a gyral crown region. To avoid this situation, we inspect $c_{\max }$ on the geodesic path. Since we know $c_{\max }$ is positive at gyral crown regions, if $c_{\max }$ at a point on the path is positive, we consider the geodesic path going through gyral crown regions and regards it as a fake sulcal fundi and discard it; otherwise, we add the geodesic path as a part of the sulcus fundi to connect breaking sulcal fundi. Figure 3 (a) shows an example of connecting breaking sulcal fundi.

Due to the numerical estimation error of curvatures and their derivatives, the extracted sulcal fundi may contain some sudden bumps as show in figure 3 (b). Therefore, we have to smooth the extracted sulcal fundi to make it more practical. 


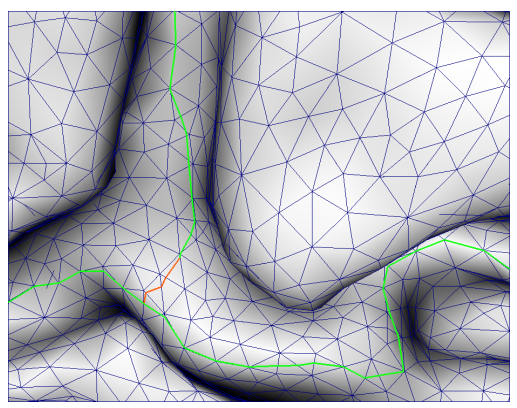

(a)

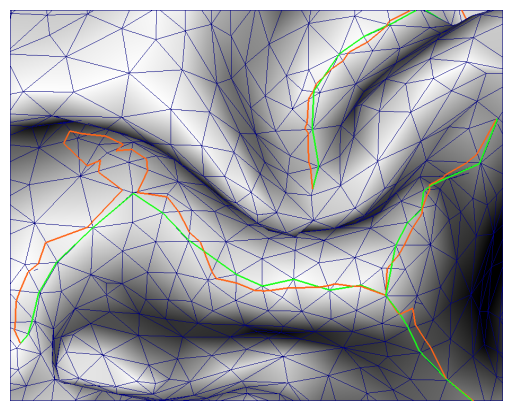

(b)

Fig. 3. Examples of connecting breaking sulcal fundi and smoothing sulcal fundi. The green curves are the original extracted sulcal fundi. In (a) the orange curve connects the breaking sulcal fundi. In (b) the orange curves are the smoothed sulcal fundi.

Meanwhile, we want to keep the smoothed sulcal fundi close to the originally extracted sulcal fundi. To handle this issue, we adopt the fast marching method to extract the weighting geodesic path from sulcal fundi starting points to the corresponding end points or junction points for smoothing and set the marching speed at each vertex as:

$$
F_{2}(\mathbf{x})=\beta \times F_{1}(\mathbf{x})+(1-\beta) \times S(\mathbf{x})
$$

Where the term $S(x)$ is used to favor vertices closing the original sulcal fundi, and $\beta$ is a parameter used to control the tradeoff between favoring $c_{\max }$ and favoring closeness to the original sulcal fundi. In the current implementation, $S(\mathbf{x})$ is set to be:

$$
S(\mathbf{x})=1-\min _{\mathbf{x}_{i}}\left(\left|\mathbf{x}-\mathbf{s}_{i}\right| /\left|\mathbf{x}-\mathbf{x}_{i}\right|\right)
$$

$\mathbf{x}_{i}$ is the one-ring adjacent vertices of $\mathbf{x}$ while $\mathbf{S}_{i}$ is the sulcal fundi point on the edge formed by $\mathbf{x}$ and $\mathbf{x}_{i}$. If no sulcal fundus point exists in edges formed by $\mathbf{x}$, $S(\mathbf{x})$ is set to be 0 . The straightforward explanation is that the closer is the current vertex to sulcal fundus point, the favorer is the current vertex in smoothing. The parameter $\beta$ is set as 0.7 in the paper. Using the marching speed calculated above, we smooth the sulcal fundi by computing the weighted geodesic path between the sulcal fundi start points and the corresponding end points or junction points. Figure 3 (b) shows an example of extracted sulcal fundi before and after smoothing.

To summarize, our new method of sulcal fundi extraction consists of four steps: estimating curvatures and their derivatives, detection of sulcal fundi segments, linking sulcal fundi segments and combining adjacent sulcal fundi, and connecting breaking sulcal fundi and smoothing. Figure 4 shows an example of extracted sulcal fundi. 


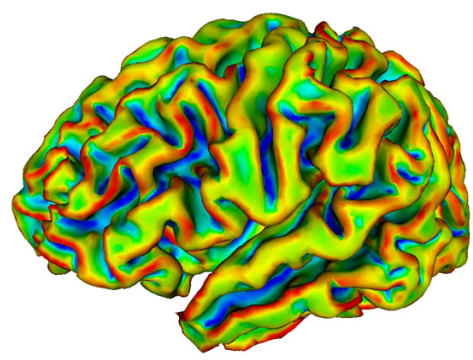

(a)

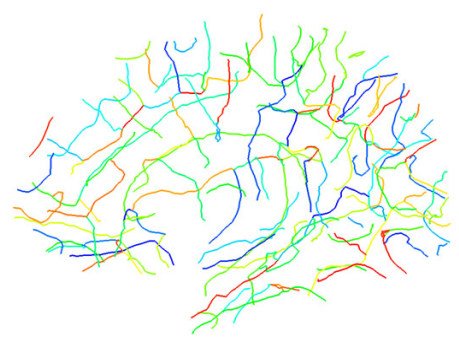

(b)

Fig. 4. An example of the extracted sulcal fundi. (a) A cortical inner surface with estimated maximum principal curvature. (b) The finally extracted sulcal fundi.

\section{Evaluation and Validation}

We tested the sulcal fundi detection method on the inner surface of 10 normal human brains. All the cortical surfaces are generated via the BrainVISA software [14]. In the inner surfaces of right hemispheres of the 10 subjects, after linking sulcal fundi segments as curves, we find 124.4 sulcal fundi connecting components on average with the standard derivation 6.7; after combining adjacent sulcal fundi curves, the number is reduced to 83.9 on average with the standard derivation 3.7 ; after connecting breaking sulcal fundi curves, the number is further reduced to 59.1 on average with the standard derivation 5.1. We have visually inspected the sulcal fundi extraction results and found no fatal errors in the 10 cases. Figure 5 shows two examples of extracted sulcal fundi on the inner surface of the right hemisphere.

To quantitatively evaluate the accuracy of sulcal fundi extraction method, we have experts manually label major sulcal fundi on the cortical inner surface of the right hemisphere of the 10 cases. We use two measurements to validate the performance. Denote the ground truth sulcal fundi as $S_{g}$ and automatic extracted sulcal fundi as $S_{a}$, the two distance measurements are defined as:

$$
\begin{aligned}
& d_{\min }=\frac{1}{n} \sum_{i \in n} \min _{j \in m}\left|S_{a}(i)-S_{g}(j)\right| \\
& d_{\max }=\max _{i \in n}\left(\min _{j \in m}\left|S_{a}(i)-S_{g}(j)\right|\right)
\end{aligned}
$$

where $n$ and $m$ are the total point numbers in sulcal fundi $S_{a}$ and $S_{g}$ respectively. $d_{\text {min }}$ measures the average distance from all the points in $S_{a}$ to the corresponding closet point in $S_{g}$. And $d_{\max }$ measures the worst maximum distance from all the points in $S_{a}$ to the corresponding closet point in $S_{g}$. We use several major sulci including central, precentral, postcentral, superior frontal, superior temporal, cingulate and calcarine sulcus to validate the performance of the method. Figure 6 shows an example of several manual labeled sulcal fundi and automatically extracted sulcal fundi on a lateral surface of a subject. For the convenience of inspection, we also 


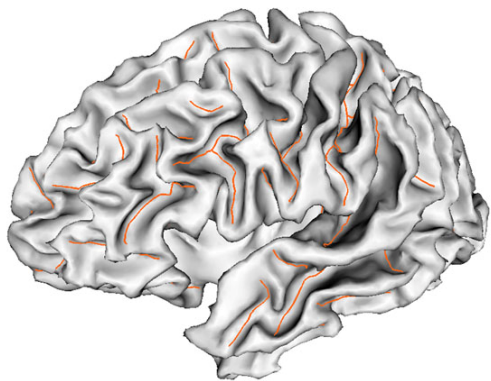

(a)

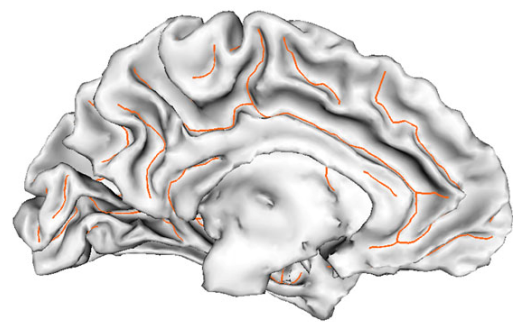

(c)

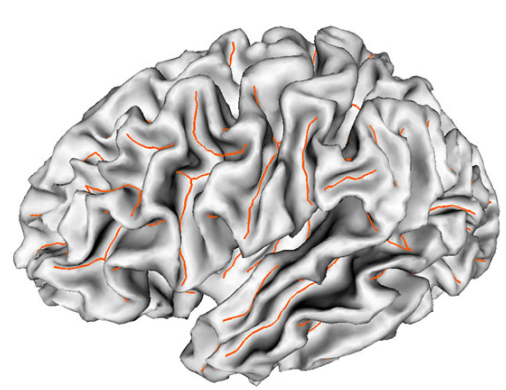

(b)

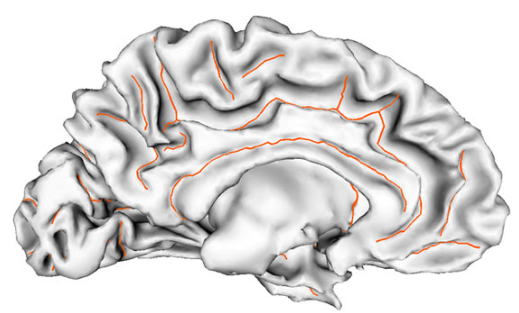

(d)

Fig. 5. Extracted sulcal fundi (orange curves) on the inner surfaces of two subjects. (a) and (b) are lateral view. (c) and (d) are medial view.

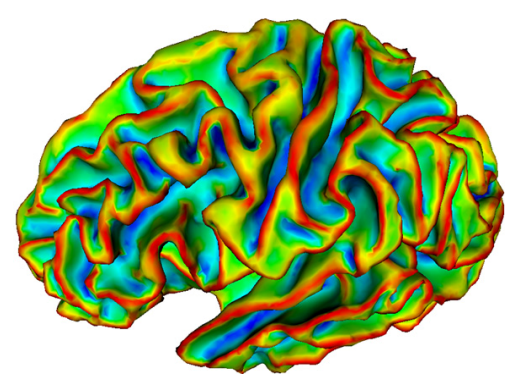

(a)

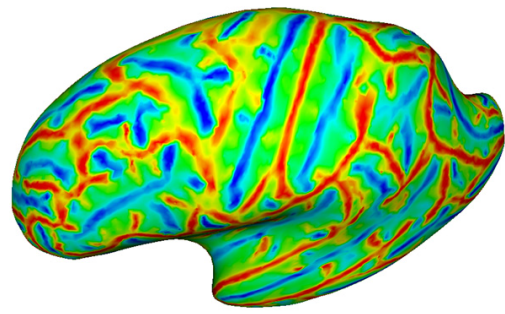

(c)

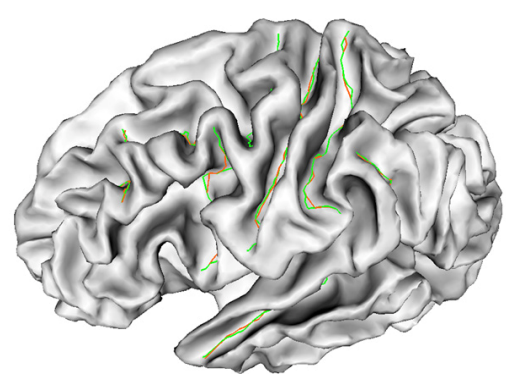

(b)

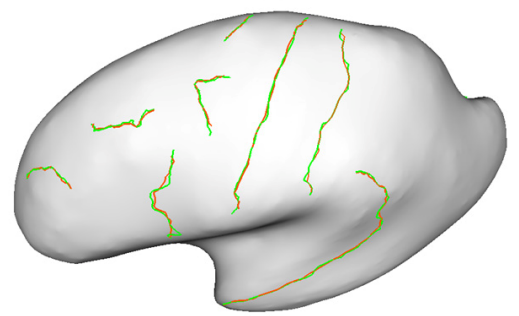

(d)

Fig. 6. An example of manual labeled and automatic extracted suclal fundi on a lateral inner surface. (a) and (c) are maximum principal curvature maps on the surface and the corresponding inflated surface. (c) and (d) are manual labeled and automatic extracted sulcal fundi overlaid on the surface and inflated surface. The green curves are automatic extracted sulcal fundi and the yellow curves are manually labeled sulcal fundi. 
overlay the sulcal fundi on the inflated surface. The inflated surface is generated via a method similar to [15]. As we can see the two sulcal fundi sets are close to each other. Table 1 shows the details of average $d_{\min }$ the several sulcal fundi on the 10 subjects. The average $d_{\min }$ is consistently less than $0.7 \mathrm{~mm}$ and the $d_{\max }$ is consistently less than $3.2 \mathrm{~mm}$, indicating the good performance of the proposed method. Currently the method is implemented using the $\mathrm{C} / \mathrm{C}++$ language. On an Intel Core $21.86 \mathrm{GHz}$ machine with 2 GB memory, it takes around 8 seconds extract all the sulcal fundi on the inner surface of a hemisphere.

Table 1. The $d_{\min }$ of several major sulcal fundi on the inner surfaces of the 10 subjects

\begin{tabular}{|c|c|c|c|c|c|c|c|}
\hline $\begin{array}{l}\text { Sulcus } \\
(\mathrm{mm})\end{array}$ & Central & $\begin{array}{l}\text { Pre- } \\
\text { central }\end{array}$ & $\begin{array}{l}\text { Post- } \\
\text { central }\end{array}$ & $\begin{array}{l}\text { Superior } \\
\text { frontal }\end{array}$ & $\begin{array}{l}\text { Superior } \\
\text { temporal }\end{array}$ & Cingulate & Calcarine \\
\hline 1 & 0.61 & 0.93 & 0.92 & 0.52 & 0.62 & 0.66 & 0.51 \\
\hline 2 & 0.66 & 0.74 & 0.49 & 0.60 & 0.60 & 0.73 & 0.56 \\
\hline 3 & 0.71 & 0.57 & 0.73 & 0.55 & 0.67 & 0.32 & 0.82 \\
\hline 4 & 0.81 & 0.57 & 0.51 & 0.41 & 0.64 & 0.30 & 0.46 \\
\hline 5 & 0.62 & 0.61 & 0.66 & 0.64 & 0.52 & 0.45 & 0.58 \\
\hline 6 & 0.71 & 0.35 & 0.78 & 0.70 & 0.65 & 0.62 & 1.16 \\
\hline 7 & 0.61 & 0.78 & 0.49 & 0.58 & 0.67 & 0.38 & 0.53 \\
\hline 8 & 0.57 & 0.64 & 0.69 & 0.42 & 0.63 & 0.35 & 0.44 \\
\hline 9 & 0.72 & 0.54 & 0.63 & 0.39 & 0.65 & 0.62 & 0.50 \\
\hline 10 & 0.78 & 0.62 & 0.49 & 0.90 & 0.61 & 0.48 & 0.46 \\
\hline Average & 0.68 & 0.64 & 0.64 & 0.57 & 0.63 & 0.49 & 0.60 \\
\hline
\end{tabular}

\section{Conclusion}

In this paper, we presented a novel automated method for accurately extracting sulcal fundi on the cortical surface of the human brain. The method has been applied to 10 normal subjects on inner surface and its performance has been evaluated using the ground truth defined by experts manually labeled sulcal fundi. The evaluation results show that the proposed method is able to extract sulcal fundi efficiently and accurately.

\section{Acknowledgement}

This study is funded by a Bioinformatics Program grant from The Methodist Hospital Research Institute to STCW.

\section{References}

1. Ono, M., Kubik, S., Abarnathey, C.: Atlas of the Cerebral Sulci. Thieme Medical Publishers (1990)

2. Lohmann, G.: Extracting line representations of sulcal and gyral patterns in MR inages of the human brain. IEEE Trans. On Medical Imaging 17(6), 1040-1048 (1998) 
3. Khaneja, N., Miller, M., Grenander, U.: Dynamic programming generation of curves on brain surfaces. IEEE Trans. On PAMI 20(11), 1260-1265 (1998)

4. Goualher, G.L., Procyk, E., Collins, D.L., Venugopal, R., Barillot, C., Evans, A.C.: Automated extraction and variability analysis of sulcal neuroanatomy. IEEE Trans. On Medical Imaging 18(3), 206-217 (1999)

5. Tao, X., Prince, J.L., Davatzikos, C.: Using a statistical shape model to extract sulcal curves on the outer cortex of the human brain. IEEE Trans. on Medical Imaging 21(5), 513-524 (2002)

6. Rivi'ere, D., Mangin, J.-F., Papadopoulos-Orfanos, D., Martinez, J., Frouin, V., R'egis, J.: Automatic recognition of cortical sulci of the human brain using a congregation of neural networks. Med. Image. Anal. 6, 77-92 (2002)

7. Rettmann, M.E., Han, X., Xu, C., Prince, J.L.: Automated sulcal segmentation using watersheds on the cortical surface. NeuroImage 15(2), 329-344 (2002)

8. Kao, C., Hofer, M., Sapiro, G., Stern, J., Rehm, K., Rottenberg, D.A.: A geometric method for automatic extraction of sulcal fundi. IEEE Trans. On Medical Imaging 26(4), 530-540 (2007)

9. Tu, Z., Zheng, S., Yuille, A.L., Reiss, A.L., Dutton, R.A., Lee, A.D., Galaburda, A.M., Dinov, I., Thompson, P.M., Toga, A.W.: Automated extraction of the cortical sulci based on a supervised learning approach. IEEE Trans. On Medical Imaging 26(4), 541-552 (2007)

10. Rusinkiewicz, S.: Estimating curvatures and their derivatives on triangle meshes. In: Proc. 3DPVT, pp. 486-493 (September 2004)

11. Ohtake, Y., Belyaev, A., Seidel, H.P.: Ridge-valley on meshes via implicit surface fitting. Proc. SIGGRAPH 23(3), 609-612 (2004)

12. Kimmel, R., Sethian, J.A.: Computing geodesic paths on manifolds. Proc. Natl. Acad. Sci. USA 95(15), 8431-8435 (1998)

13. Tao, X., Han, X., Rettmann, M., Prince, J., Davatzikos, C.: Statistical study on cortical sulci of human brains. In: Insana, M.F., Leahy, R.M. (eds.) IPMI 2001. LNCS, vol. 2082, pp. 475-487. Springer, Heidelberg (2001)

14. Cointepas, Y., Mangin, J.F., Garnero, L., Poline, J.B., Benali, H.: BrainVISA: Software platform for visualization and analysis of multimodality brain data. NeuroImage 13, S98 (2001)

15. Tosun, D., Rettmann, M.E., Prince, J.L.: Mapping techniques for aligning sulci across multiple brains. Med. Image. Anal. 8, 295-309 (2004)

16. Thirion, J.-P.: The extermal mesh and understanding of $3 \mathrm{D}$ surfaces. International Journal of Computer Vision 19, 115-128 (1996) 